\title{
The Guttmann Institute of Barcelona
}

\author{
M Sarrias ${ }^{1}, \mathrm{JM}$ Ramirez ${ }^{2}$ and $\mathrm{J} \mathrm{Vidal}^{3}$ \\ ${ }^{1}$ Former Medical Director and Founder, ${ }^{2}$ General Manager, ${ }^{3}$ Head Clinician, Instituto Guttmann, Garcilaso 57, 08027 \\ Barcelona, Spain
}

\begin{abstract}
The Guttmann Institute is a hospital specialised in the comprehensive rehabilitation and medical/surgical treatment of persons suffering from spinal cord injury or from other severe physical disabilities. It opened in Barcelona in 1965 and was the first hospital in Spain dedicated to the treatment of paraplegic and tetraplegic patients, and the one which introduced into Spain the comprehensive approach to spinal cord injury developed by Sir Ludwig Guttmann at the Stoke Mandeville Hospital in the UK. The Guttmann Institute Foundation is a non-profit-making foundation and its hospital, the Guttmann Institute, forms part of the Public Service Hospital Network. The Guttmann Institute has a wide-ranging, multi-disciplinary group of staff made up of specialised professionals; and it has the appropriate resources for the provision of thorough medical attention from a technical, scientific and human point of view.
\end{abstract}

Keywords: spinal cord injuries hospital; staff; rehabilitation; medical services; functional programmes

\section{History}

The Guttmann Institute Foundation was founded in Barcelona in 1962 by Mr González Gilbey who, on becoming a tetraplegic after a car accident in 1958, had personal experience of the serious consequences arising from the non-existence in Spain of centres specialised in the treatment of persons suffering from spinal cord injuries.

In order to obtain the appropriate medical/ rehabilitation treatment, $\mathrm{Mr}$ González Gilbey was transferred to the National Spinal Injuries Centre at Stoke Mandeville Hospital in England where he was able to achieve the necessary rehabilitation to allow him to be his energetic former self, in spite of his great physical limitations and his dependence on a wheelchair. On returning to Barcelona, he set himself the objective of creating a specialised medical centre capable of providing his fellow Spanish paraplegia and tetraplegia sufferers with the kind of medical and rehabilitation treatment he had received abroad.

His enthusiasm for the project together with his strong will made it possible that on the 27th November 1965, the first hospital in Spain dedicated to the treatment and comprehensive rehabilitation of persons suffering from spinal cord injury was inaugurated in Barcelona (Figure 1).

The new hospital was named the Guttmann Institute in honour of Sir Ludwig Guttmann who, in the face of the bleak prognosis for victims of spinal cord injuries, established the renovative principles for their treatment and rehabilitation at Stoke Mandeville

Correspondence: M Sarrias
Hospital, during the second World War. Dr Guttmann's contribution was so decisive in changing that bleak prognosis, that medical history distinguishes between paraplegia before and after his intervention.

To form the new hospital's technical team, $\mathrm{Mr}$ González Gilbey was able to count on one of Sir Ludwig Guttmann's disciples - who has been medical director of the Guttmann Institute Hospital for 32 years - together with a nurse and physiotherapist also trained at Stoke Mandeville. These three professionals formed the nucleus from which a staff of over 260 people has evolved.

Housed in what was formerly a Venereal Disease Hospital, the building has been remodelled to allow it to offer the adequate service of it's new function. It has 96 beds and, up to 27th November 1997, more than 5000 in-patients have received treatment and rehabilitation in this hospital.

The Guttmann Institute Hospital forms part of the Public Service Hospital Network and is classified by the 'Catalan Health Service' as a Referral Hospital for the provision of specialised care to beneficiaries of the Public Health Care System (Social Security and other affiliated organisations). In addition, it has made agreements with the principal Mutual Work Injury Insurance Companies, other insurance companies which cover the risks derived from traffic accidents and with the major private health care organisations.

The adoption of a policy of permanent improvement, based on research, continuous staff training and the exchange of knowledge and experience with other professionals and medical centres, has allowed the Guttmann Institute to retain its position as a pioneer 


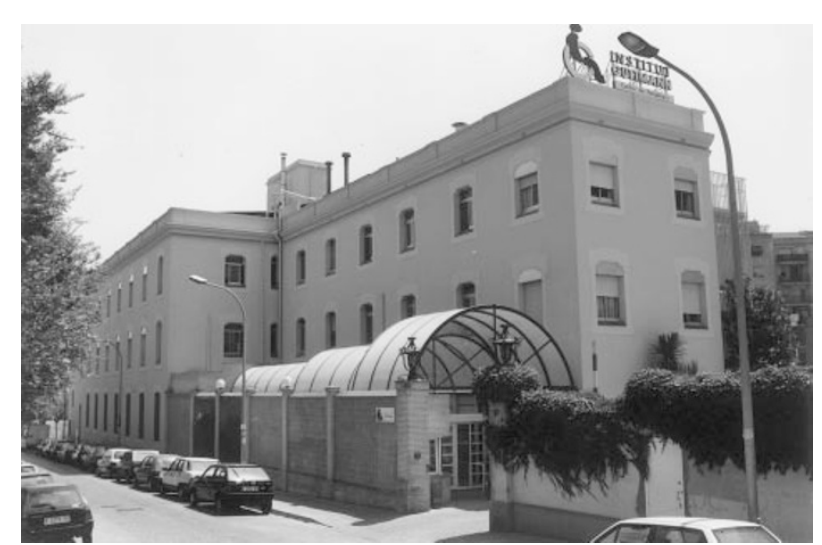

Figure 1 The Guttmann Institute Hospital in Barcelona

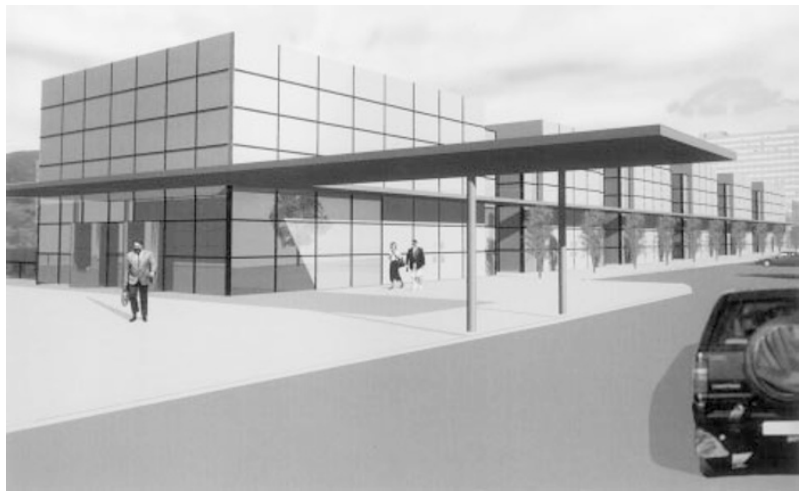

Figure 2 Model view of the new Guttmann Institute hospital (teaching general hospital in background) in the introduction of new therapeutic tools for improving the quality of life of persons suffering from spinal cord injury and other severe physical disabilities.

In order to provide an adequate response to a growing and increasingly complex demand, the Guttmann Institute Foundation promoted and established a co-operation agreement with the Catalan Health Service and the ONCE Foundation (a Foundation to help persons with any type of disability within the National Organisation for the Blind) for the financing and construction of a new hospital building within the grounds of a teaching general hospital in Badalona (town adjacent to the north side of Barcelona) (Figure 2).

This new hospital with a built area of 16000 square metres, including a sports pavilion, is due to be finished in the year 2000. It will allow Guttmann Institute Foundation to meet the health care challenges which lie ahead in the coming decades. The current building will then be used as an Assisted Residence and Day Centre, to provide specific responses to the social and health care needs of persons with established severe physical disabilities.

\section{Spinal cord injuries}

A spinal cord injury always represents a major upset in the life of the person involved, both from a physical and psychological as well as from a social point of view. The specialised treatment and rehabilitation of this group of people makes it possible for contact and interaction to take place between recently injured patients, those who are already veteran paraplegics and tetraplegics, their respective families, the specialised professionals and the community at large, giving rise to the creation of a specific spinal cord injury culture which increases experience and facilitates care improvement of these patients. At the same time, through the dissemination of information to society as a whole, social awareness of this group of people is awakened and this helps to make the accident prevention campaigns, particularly for the road traffic accidents, more meaningful. Since 1990 the Guttmann Institute is running a spinal cord and head injury prevention campaign, under the name of 'No more blows', addressed to the 14 to 18 year-old boys and girls at their schools; it is presented by young spinal cord injured volunteers and so far 40000 pupils of some Barcelona schools have attended these sessions.

Medical/Surgical and Rehabilitation treatment of persons with spinal cord lesions is by far $(72 \%$ of its patients) the main concern of Guttmann Institute. Out of a total of 530 admissions in 1996, 381 patients had spinal cord lesion; 270 with paraplegia and 111 with tetraplegia. The average hospitalisation time for comprehensive treatment and rehabilitation was 129 days.

About $80 \%$ of the Hospital's spinal cord injury patients are transferred from the large general hospitals in Barcelona and in the rest of Catalonia (a region in the north-east corner of mainland Spain which has an extension of 32000 square kilometres and a population of 6000000 inhabitants). The remaining $20 \%$ come from outside Catalonia. Most of these patients arrive within the first 3 weeks of the onset of the spinal cord injury or disease.

The comprehensive rehabilitation programmes of Guttmann Institute aim at having a positive influence on the aptitudes and attitudes of its patients so that they may eventually return to the community as active, responsible and caring citizens. These programmes include: treatment and rehabilitation of patients with spinal cord injuries or disease from admittance up to the achievement of the maximum possible level of self-sufficiency for a successful social resettlement. Personalised follow-up treatment by means of regular check-ups to prevent the complications specific to the disability. Psychological and social follow-up of persons subsequent to their discharge. General information and personal assessment concerning new procedures, up-to-date techniques and latest 
developments which enhance the health and quality of life of persons with spinal cord injury. Specialised treatment of all medical/surgical problems which might occur during the entire lifetime of the patient (Figure 3).

The Guttmann Institute Interdisciplinary Rehabilitation Team is responsible for implementing these programmes and for achieving the active participation of the patients themselves and their respective families. This interdisciplinary team is made up of the various professionals: the nurse, the occupational therapist, the physiotherapist, the social worker, the psychologist and the Paraplegia Specialist Doctor who acts as coordinator of the team and is the person responsible for the patient's programme during the entire period of his or her stay in the hospital. The rehabilitation team is responsible for determining the therapeutic objectives in each case, evaluating the degree of rehabilitation achieved and deciding on discharge from hospital.

The Guttmann Institute professional team is distributed amongst the following areas:

Medical area. It is made up of doctors with different specialities who have chosen to train specifically for this type of pathology. In addition, it has at its disposal all the necessary medical and surgical specialities to complete the process of diagnosis and therapy for patients with spinal cord injury or any other major physical disability.

Nursing area. This area has responsibility for integral nursing care, incorporating rehabilitative objectives into the completion on their nursing tasks and the day-to-day activities of the patients. It motivates and guides patients to do as much as possible for themselves, thereby improving personal skills and self-sufficiency. It is also responsible for instilling in patients the habits and techniques of self care and the prevention of complications, at the same time as providing training for relatives or other persons close to the patient. Both the qualified nursing staff and hospital auxiliaries establish an important supportive relationship with patients, have first-hand experience of their progress and emotional state, and maintain constant communication with other members of the rehabilitation team as well as with the families or other persons with whom the patient has close emotional ties (Figure 4).

Psycho-social area. This area is made up fundamentally of clinical psychologists, neuro-psychologists and social workers and is responsible for the assessment, guidance and follow-up of the social and psychological aspects. It provides emotional support to the patient and the family and fosters the progressive re-establishment of contact between the patient and society. It offers guidance and individual, family and group attention, providing neuro-psychological treatment for those patients with cognitive alterations and logopedic treatment for those with speech disorders. This team also promotes the internal and participative activities carried out at the hospital, together with others which stimulate and facilitate social interaction. The colla-

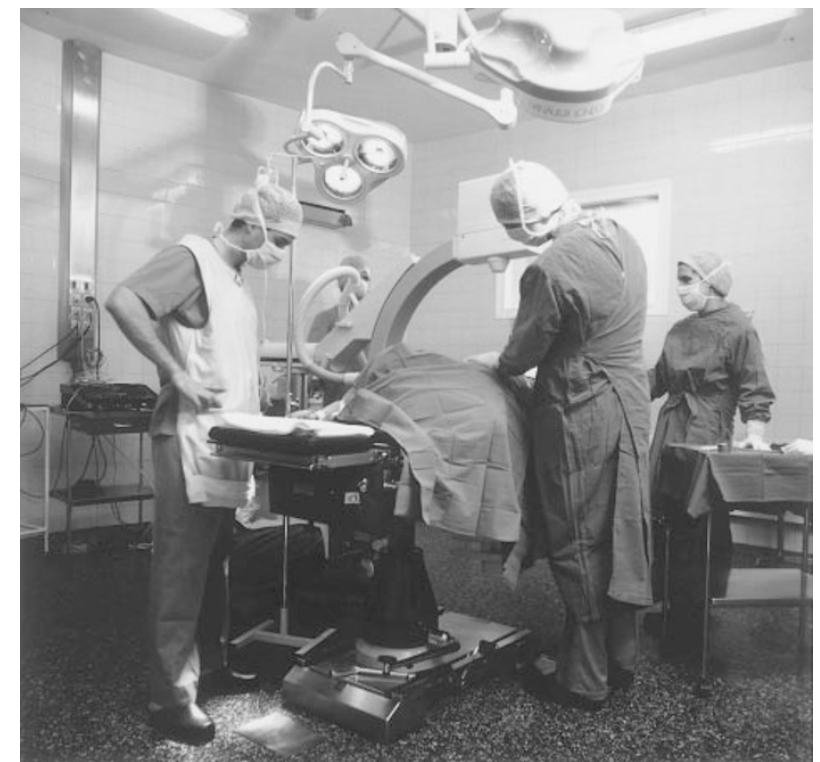

Figure 3 Main operating theatre (setting a fractured femur in a paraplegic patient)

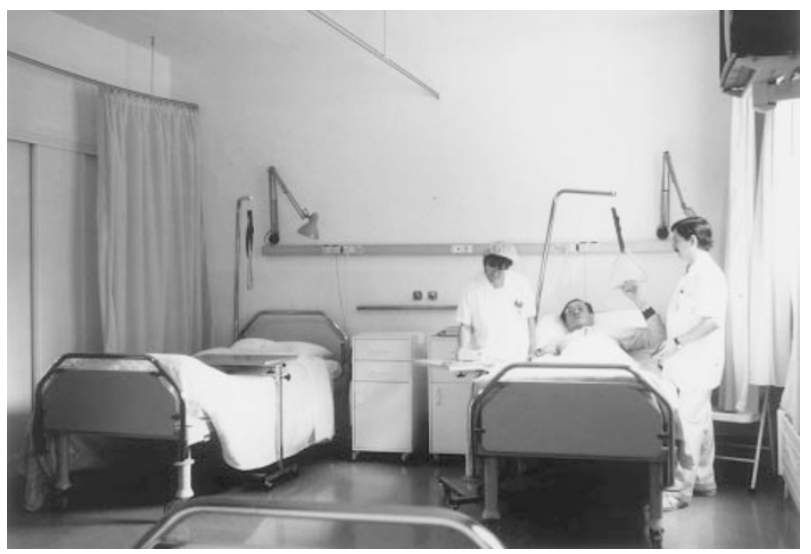

Figure 4 Four bedded room in one of the three hospital wards

boration of veteran spinal cord injury sufferers and social work volunteers towards this aim is also of value.

Functional rehabilitation area. This area is made up of occupational therapists, physiotherapists and auxiliary staff, all of them specialised in the treatment of major physical disabilities and, in particular, spinal cord injury. It has the support of orthopaedic appliance technicians for the fitting of ortho-prosthetic devices. It is responsible for achieving for each patient the highest possible degree of self-sufficiency in carrying out his or her day-to-day activities and maintaining social relationships. It also provides the patient with fitness training, including sport in its recreational or competitive aspects (Figures 5, 6 and 7). 


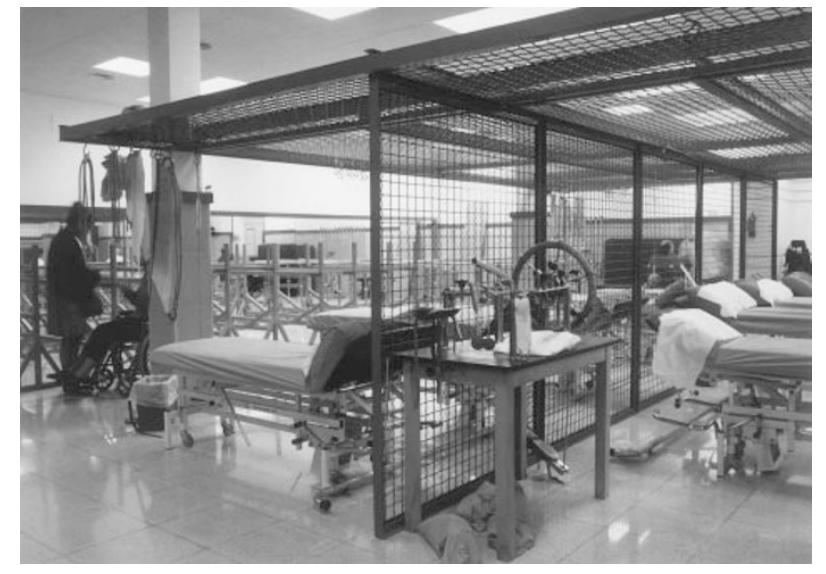

Figure 5 Partial view of physiotherapy department

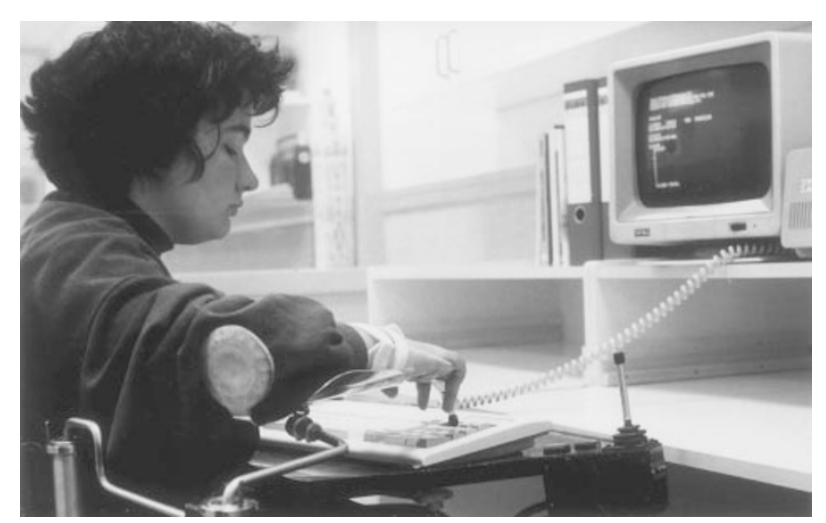

Figure 6 A below $\mathrm{C} 4$ tetraplegic patient in occupational therapy

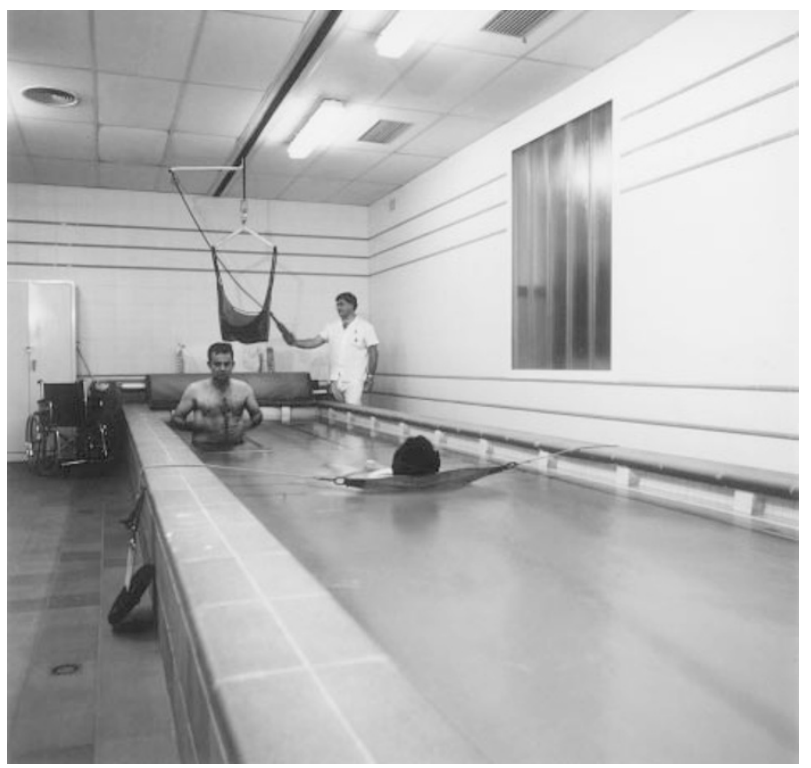

Figure 7 Hydrotherapy
Table 1 shows the total numbers of Guttmann Institute personnel and their equivalent numbers if each person worked full-time.

\section{Learning to live with a spinal cord injury}

Becoming accustomed to the use of a wheelchair and, if possible, learning how to walk again with calipers and walking sticks or between parallel bars, requires effort and perseverance. Normal daily activities such as eating, getting dressed or washing are, at first, difficult and demanding tasks. Facing up to the human, emotional and social consequences of paraplegia or tetraplegia is not something to be achieved in a day. It is essential, therefore, to establish a relationship of trust between the patient and the rehabilitation team.

When the patient and his or her family become aware of the disability and are unexpectedly confronted with an undesired reality, the hospital staff provide the guidance and emotional support so necessary at these particularly difficult moments, helping them to cope with the changes which this new physical situation involves. Also, the exchange of experience with other more veteran patients is usually a positive influence towards this aim.

Throughout the hospitalisation period, the patient, in collaboration with the rehabilitation team and with the help of informative and audio-visual training material published by the Guttmann Institute, obtains skills and confidence to carry out those activities of daily living compatible with the level and extension of the spinal cord lesion. The patient is introduced to sporting activities such as basketball, swimming, archery, table tennis, tennis and athletics, and has the opportunity to join in group excursions and to participate in socio-cultural and re-socialisation pro-

\section{Table 1}

\begin{tabular}{lcc}
\hline Staff & $\begin{array}{c}\text { Number of } \\
\text { persons }\end{array}$ & $\begin{array}{c}\text { Full-time } \\
\text { equivalence }\end{array}$ \\
\hline Doctors & 36 & 16.8 \\
Psychologists & 4 & 3.1 \\
Pharmacists & 2 & 1.1 \\
Staff nurses & 55 & 40.8 \\
Physiotherapists & 13 & 9.8 \\
Occupational therapists & 6 & 5.2 \\
Social workers & 3 & 2.6 \\
Auxiliary nurses (male) & 62 & 48.6 \\
Auxiliary nurses (female) & 23 & 14.8 \\
Directive staff & 4 & 3.5 \\
Administrative staff & 24 & 18.8 \\
General services & 29 & 26.8 \\
Total & 261 & 191.9 \\
\hline
\end{tabular}

(Catering and laundry services are sub-contracted) 
grammes. Assistance is given to re-convert a driving license or to obtain a new one. Going home at weekends is stimulated as soon as the patient's condition so allows, in order to re-establish contact with the previous environment and to prepare returning home under the new reality (Figures 8 and 9).

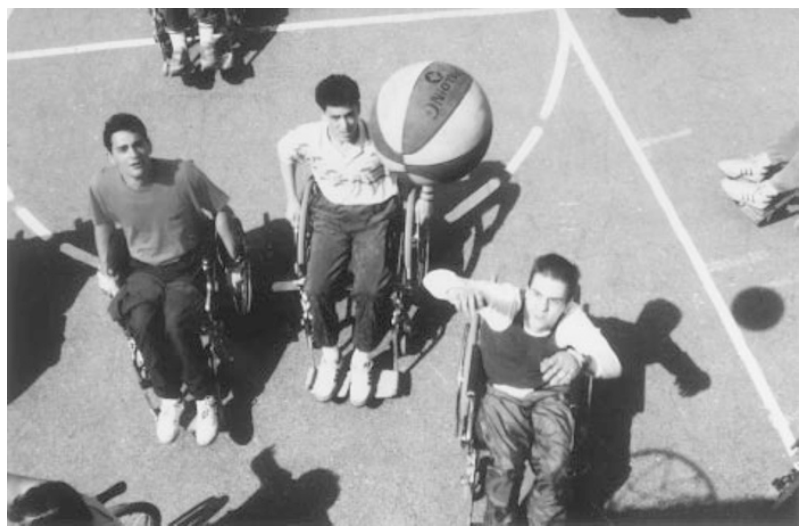

Figure 8 Therapeutic sport on the Guttmann Institute basketball court

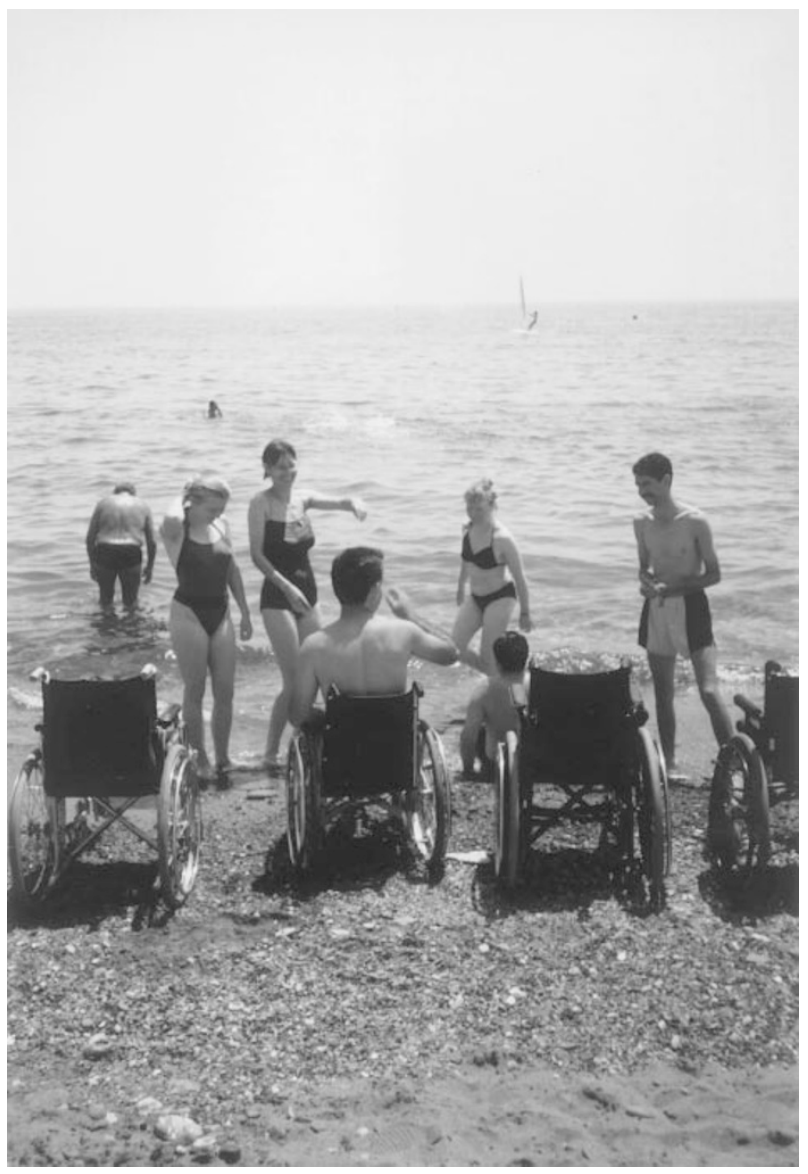

Figure 9 Weekly group outing organised by the Guttmann Institute
Long before discharge from hospital, patient, family and rehabilitation team get together to organise the return home. The importance of preventing complications and maintaining a good health is stressed and information is provided on technical aids, home adaptations, mobility, sexuality, social services and the possibility to return to professional life. Once at home, the disabled person knows that the Guttmann Institute professional team will help to solve any difficulties that might appear and will suggest solutions and alternatives for discussion. The social integration studies carried out on discharged patients show that, with time, the majority feel satisfied with their new lifestyle, with a good number of them indulging in full or part time employment, and a large proportion of the younger patients starting their own families.

\section{Medical services and functional programmes}

The Guttmann Institute hospital, founded specifically for the provision of medical attention to persons suffering from spinal cord injury has, through time, expanded its services in order to offer an appropriate response to the growing demand for the treatment of other major physical disabilities.

Today, the Guttmann Institute provides comprehensive rehabilitation and specialised medical/surgical treatment to adults and children suffering from: spinal cord injury and disease, spina bifida, multiple sclerosis, head injuries, and other neurological syndromes which have potentially disabling consequences.

The medical and rehabilitation care undertaken at the Guttmann Institute is recorded in protocols and carried out by means of programmes. In addition to the general or basic aspects of comprehensive rehabilitation, specific functional programmes are implemented to bring about qualitative improvements in the various areas of the health and degree of adaptation of sufferers of major physical disabilities. Noteworthy among these are: spinal surgery to reduce and stabilise potentially unstable fractures, though most reduction and stabilisation of vertebral fractures is effected conservatively by means of postural reduction on kinetic beds. Upper limb surgery to provide a key or finger grip to tetraplegic hands. Sacral anterior roots electro-stimulation (Professor Brindley's SARS implant used in Guttmann Institute since 1990 with a variation known as the 'Barcelona Technique') for bladder control, in some patients bowel control as well and, in the case of most men, also for implant driven erection. Pain-control and spasticity clinic for assessment, treatment and follow-up of pain and spasticity, implanting peridural stimulators and infusion sets for intrathecal administration of medication when necessary. Treatment and rehabilitation of sexual function, male fertility and obstetric-gynaecological care. Functional electrical stimulation (FES) programmes with surface electrodes for standing and therapeutic walking exercise were introduced in 1991. 
Self-sufficiency and control of immediate environment programme. Rehabilitation for children outside school hours to make compatible regular schooling with outpatient rehabilitation to children with major physical disabilities (daily from 5 to $7 \mathrm{pm}$ ). Relaxation and biofeedback programmes as a complement for the treatment of stress, pain and anxiety, carried out by the clinical psychology department.

The Guttmann Institute provides its health-care services by means of: Hospitalisation. For this it has at its disposal 96 hospital beds and an adapted flat from a block close to the hospital which allows the patient to experience the level of self-sufficiency achieved in more private and real life surroundings; Out-patient rehabilitation. For patients with a fair degree of selfsufficiency living within the city of Barcelona. It operates as a day hospital from 10 am to $5 \mathrm{pm}$ with 30 places available; Out-patient consultations by appointment. The Guttmann Institute provides its services to all persons without any form of discrimination on grounds of age, race, sex, religion, beliefs, status, origins or nationality. 